\title{
Observer-based Feedback Control for Stabilization of Collective Motion
}

\author{
Seth Napora and Derek A. Paley
}

\begin{abstract}
Collective motion of a multi-vehicle testbed has applications in weather monitoring and ocean sampling. Previous work in this field has produced theoretically justified algorithms for stabilization of parallel and circular motions of self-propelled particles using measurements of relative position and relative velocity. This paper describes an observer-based feedback algorithm for stabilization of parallel and circular motions using measurements of relative position only. This algorithm utilizes information about the particle dynamics and turning rates to estimate the relative velocities. We describe a laboratory-scale underwater vehicle testbed on which the algorithm is being implemented.
\end{abstract}

\section{INTRODUCTION}

Motivation for pursuing coordinated, collective motion of autonomous vehicles comes partly from biology. From ants that collectively build their colonies to fish that school as one unit for defensive and other purposes, it is apparent that collective behavior is a beneficial component to many groups. Although animals may utilize collective behavior to achieve various results, multiple members help to reduce the workload on each individual member.

Current study in the field of collective motion has produced various results. Researchers have extended the selfpropelled particle model used in [7] to handle more situations. In [6], control laws for self-propelled particles were proven to stabilize synchronized, balanced, circular, and symmetric circular formations in the presence of a timeinvariant flowfield. Taking another direction, [5] provided a rotational acceleration controller for a self-propelled particle using backstepping and proportional control. Another method for creating collective motion utilized pursuit dynamics. In [3], this concept was examined whereby a leader particle performed a behavior and the others pursued the leader.

Another area of interest in the field of collective motion concerns the stabilization of particle formations with limited information. [9] discussed a flocking behavior of agents whereby only a certain number of agents were informed of the desired behavior. Flocking motion was achieved under this restriction, as in [8], which discussed a self-propelled particle system with limited communication between agents. Another form of limited information can be a result of limited sensing capabilities. One way to solve this problem is

This material is based upon work supported by the National Science Foundation under Grant Nos. CMMI0928416 and CMMI0954361 and the Office of Naval Research under Grant No. N00014-09-1-1058.

S. Napora is a graduate student in the Department of Aerospace Engineering, University of Maryland, College Park, MD 20742, USA snaporalumd. edu

D. Paley is an assistant professor in the Department of Aerospace Engineering, University of Maryland, College Park, MD 20742, USA dpaleyeumd.edu by estimating the unknown variable. [2] used this approach for formation tracking utilizing sliding-mode estimators.

An additional field of research discusses real world application of collective motion in order to demonstrate the performance of a control algorithm. In order to study collective behaviors of particles, an effective testbed for analyzing control algorithms consists of identical vehicles capable of performing the control law. [1] described a testbed of LEGO MINDSTORMS that produced parallel and circular motion of vehicles around virtual beacons. The work described below focuses on a laboratory scale testbed of underwater vehicles for which inter-vehicle sensing is limited.

Parallel and circular motion has been achieved in selfpropelled particles with either first- or second-order rotational dynamics. These models have assumed that every particle is aware of the relative velocity of every other particle in the group. Here, we do not assume that each particle can sense relative velocities, only relative positions. The contribution of this paper is to present theoretically justified methods for (1) estimating the velocity of one particle relative to another particle and (2) utilizing that estimate in an observer-based feedback control to stabilize parallel and circular formations. We provide simulations to illustrate the performance of the estimation and control algorithms.

The outline for the paper is as follows. In Section II, kinematic and dynamic models of self-propelled particle motion are described, including control laws that stabilize parallel and circular formations using relative position and relative velocity. In Section III, an observer-based feedback control is derived using knowledge of relative position and turning rate. Section IV describes an underwater vehicle testbed that is being developed to demonstrate how this control and estimation will function on a physical system. Lastly, Section V summarizes the results and ongoing work.

\section{BACKGROUND}

In our study of collective motion, we consider parallel and circular formations to be building blocks for more complex motion. These cooperative motions have been achieved in [7] using a particle model to represent each vehicle in a group. We describe that model here, along with a dynamic model that includes second-order rotational dynamics. For each model, we include a description of parallel and circular control algorithms.

\section{A. First-Order Particle Model}

A dynamic model that can be used to design collective motion is a first-order particle model. This model assumes 
that each particle moves at a constant speed equal to one. The position of particle $k$ is $\mathbf{r}_{k}=\left(x_{k}, y_{k}\right)$, and the orientation of its (planar unit) velocity is $\theta_{k}$. The steering control, $u_{k}$, is applied to the heading rate allowing the vehicle to change course as indicated below:

$$
\begin{aligned}
\dot{x}_{k} & =\cos \left(\theta_{k}\right) \\
\dot{y}_{k} & =\sin \left(\theta_{k}\right) \\
\dot{\theta}_{k} & =u_{k},
\end{aligned}
$$

where $k$ represents the $k$ th particle in a group of size $N$. Collective control laws have been designed for this model resulting in parallel and circular formations.

A parallel formation is achieved when each particle has the same velocity orientation. The following control achieves this motion with all-to-all communication [7]:

$$
u_{k}=-\frac{K}{N} \sum_{j=1}^{N} \sin \left(\theta_{j}-\theta_{k}\right) \triangleq \phi_{k}(\boldsymbol{\theta}),
$$

where $\boldsymbol{\theta}=\left[\theta_{1}-\theta_{k}, \ldots, \theta_{N}-\theta_{k}\right]$. Note that the absolute orientations of the particle's velocities are not required for control, but only the relative orientation.

Choosing $K<0$ in (2) will produce straight-line motion where all the particle trajectories are parallel [7]. Choosing $K>0$ yields balanced motion; this behavior occurs when the sum of all particle velocities is equal to zero. Both motions are illustrated in Fig. 1.
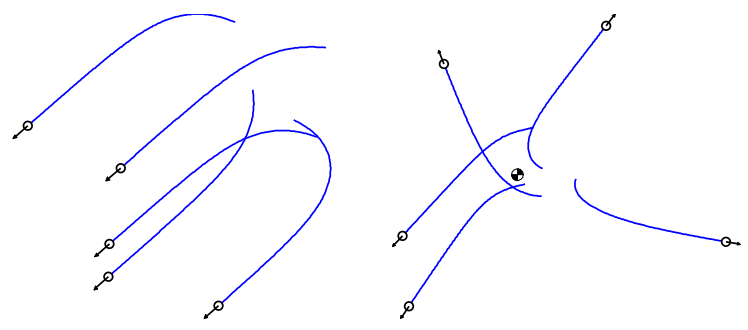

Fig. 1. Parallel motion (left) and balanced motion (right), where the particle centroid is fixed.

A circular formation is achieved when each particle's turning rate and center of rotation are identical to the rest of the group. The center of rotation can be defined in complex notation as [7]

$$
\mathbf{c}_{k}=\mathbf{r}_{k}+i \omega_{0}^{-1} e^{i \theta_{k}} .
$$

where $\mathbf{r}_{k}$ is the complex position and $\omega_{0}^{-1}$ is the circle's radius. Using the center of rotation, the following control produces a circular formation with all-to-all communication [7]

$$
u_{k}=\omega_{0}\left(1+K\left\langle P_{k} \mathbf{c}, e^{i \theta_{k}}\right\rangle\right) \triangleq \psi_{k}(\mathbf{r}, \boldsymbol{\theta}),
$$

where $\mathbf{c}=\left[\mathbf{c}_{1}, \ldots, \mathbf{c}_{N}\right]^{T}, \mathbf{r}=\left[r_{1}-r_{k}, \ldots, r_{N}-r_{k}\right]$, and $K>0 . P_{k}$ is the $k$ th row of the projector matrix $P=$ $I_{N \times N}-\frac{1}{N} \mathbf{1 1}^{T}$, where $\mathbf{1}=[1, \ldots, 1]^{T} \in \mathbb{R}^{N}$. This formation is illustrated in Fig. 2.

The circular control law can be expressed in terms of the particle's relative velocity orientations, $\boldsymbol{\theta}$, and the particle's relative positions, $\mathbf{r}$.

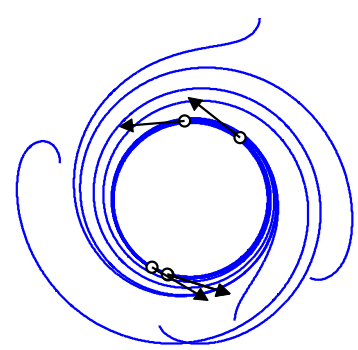

Fig. 2. A simulation of five particles performing the circular control law with random initial conditions.

\section{B. Second-Order Particle Model}

The first-order model is useful for studying various group behaviors, but may not adequately represent the rotational dynamics of an actual vehicle. Instead of controlling the heading rate to change direction, a vehicle applies a moment to control the rotational acceleration. Under this assumption, each particle has the following dynamics:

$$
\begin{aligned}
\dot{x}_{k} & =\cos \left(\theta_{k}\right) \\
\dot{y}_{k} & =\sin \left(\theta_{k}\right) \\
\dot{\theta}_{k} & =\omega_{k} \\
\dot{\omega}_{k} & =u_{k} .
\end{aligned}
$$

The control laws created for the first-order model can be extended to the second-order model using a proportional controller that drives the desired heading to the first-order control laws. The parallel formation for this model becomes [5],

$$
u_{k}=K_{p}\left(\phi_{k}(\boldsymbol{\theta})-\omega_{k}\right),
$$

where $\phi_{k}(\boldsymbol{\theta})$ is defined in (2) and $K_{p}>0$. Similarly, circular motion can be achieved using the following control law [5]

$$
u_{k}=K_{p}\left(\psi_{k}(\mathbf{r}, \boldsymbol{\theta})-\omega_{k}\right),
$$

where $\psi_{k}(\mathbf{r}, \boldsymbol{\theta})$ is defined in (4) and $K_{p}>0$. The collective behaviors produced by the first-order model are also exhibited in this extended model.

\section{THEORETICAL RESULTS}

As mentioned previously, parallel and circular motion have been achieved with both the first- and second-order models. These models have assumed that every particle is aware of the relative position and relative velocity of every other particle in the group. Here we assume knowledge of relative position and turning rate only.

\section{A. Dynamic Model}

Without loss of generality, we begin by examining a pair of particles $j$ and $k$. Fig. 3 shows particles $j$ and $k$ in an inertial frame, $\mathcal{I}$. Each particle's position relative to the origin is represented by the vectors $\mathbf{r}_{j}$ and $\mathbf{r}_{k}$, respectively, while the vector between the particles is represented by $\mathbf{r}_{j / k}=\mathbf{r}_{j}-\mathbf{r}_{k}$.

An inertial-frame representation is not necessarily known to each particle. Particle $k$ views the world from its own path frame $\mathcal{B}_{k}=\left(k, \mathbf{x}_{k}, \mathbf{y}_{k}, \mathbf{z}_{k}\right)$ which moves with the particle itself. $\mathbf{x}_{k}$ is aligned with $\dot{\mathbf{r}}_{k}$ as shown in Fig. 3 and $\mathbf{y}_{k}=$ 


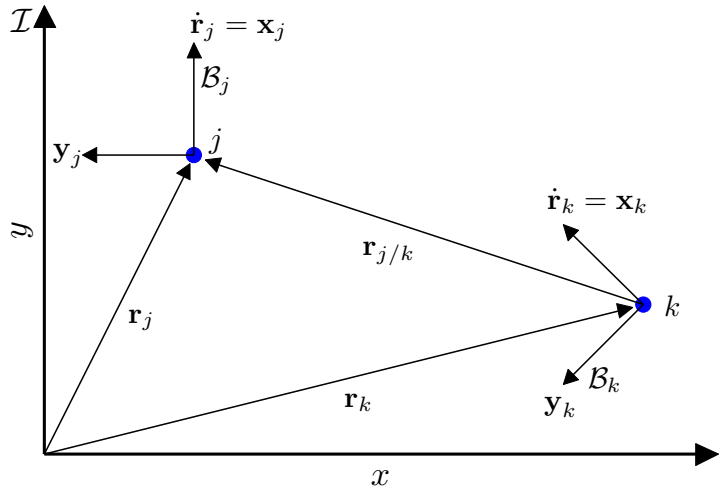

Fig. 3. Vectors utilized in dynamic model

$\mathbf{z}_{k} \times \mathbf{x}_{k}$, where $\mathbf{z}_{k}$ is out of the page. We express $\mathbf{r}_{j / k}$ as components in frame $\mathcal{B}_{k}$ as $\mathbf{r}_{j / k}=x_{j / k} \mathbf{x}_{k}+y_{j / k} \mathbf{y}_{k}$.

We begin by considering the inertial kinematics of $j$ relative to $k$. Taking the derivative of $\mathbf{r}_{j / k}$ with respect to the inertial frame and expressing the result in matrix notation with respect to frame $\mathcal{I}$ yields

$$
\begin{aligned}
{\left[{ }^{\mathcal{I}} \mathbf{v}_{j / k}\right]_{\mathcal{I}} } & =\left[\frac{{ }^{\mathcal{I}}}{d t} \mathbf{r}_{j / k}\right]_{\mathcal{I}}=\left[\dot{\mathbf{r}}_{j}-\dot{\mathbf{r}}_{k}\right]_{\mathcal{I}} \\
& =\left[\begin{array}{c}
\cos \theta_{j}-\cos \theta_{k} \\
\sin \theta_{j}-\sin \theta_{k}
\end{array}\right]_{\mathcal{I}}
\end{aligned}
$$

In this equation, ${ }^{\mathcal{I}} \mathbf{v}_{j / k}$ represents the velocity of particle $j$ with respect to $k$ in the inertial frame. The subscript $\mathcal{I}$ refers to the coordinate system in which this quantity is expressed. For example, $\left[{ }^{\mathcal{I}} \mathbf{v}_{j / k}\right]_{\mathcal{I}}$ means that the velocity of particle $j$ with respect to particle $k$ is expressed as vector components in the inertial frame.

The inertial kinematics do not contain the relative orientation, $\theta_{j}-\theta_{k}$, which is needed to implement either controller (2) or (4). To obtain the relative orientation, we rewrite the inertial velocity in particle $k$ 's path frame. The angular velocity of $\mathcal{B}_{k}$ with respect to $\mathcal{I}$ is ${ }^{\mathcal{I}} \boldsymbol{\omega}^{\mathcal{B}_{k}}=\omega_{k} \mathbf{z}_{k}$. The velocity in the inertial frame can be expressed as components in frame $\mathcal{B}_{k}$ using a $2 \times 2$ rotation matrix $R$ to rotate by $-\theta_{k}$ :

$$
\begin{aligned}
{\left[{ }^{\mathcal{I}} \mathbf{v}_{j / k}\right]_{\mathcal{B}_{k}} } & =R\left(-\theta_{k}\right)\left[{ }^{\mathcal{I}} \mathbf{v}_{j / k}\right]_{\mathcal{I}} \\
& =\left[\begin{array}{cc}
\cos \left(\theta_{k}\right) & \sin \left(\theta_{k}\right) \\
-\sin \left(\theta_{k}\right) & \cos \left(\theta_{k}\right)
\end{array}\right]\left[\begin{array}{c}
\cos \theta_{j}-\cos \theta_{k} \\
\sin \theta_{j}-\sin \theta_{k}
\end{array}\right]_{\mathcal{I}} \\
& =\left[\begin{array}{c}
\cos \left(\theta_{j}-\theta_{k}\right)-1 \\
\sin \left(\theta_{j}-\theta_{k}\right)
\end{array}\right]_{\mathcal{B}_{k}} .
\end{aligned}
$$

Although the resulting matrix contains the desired relative orientation, the term on the left is not directly measurable from the path frame. It can be related to the path frame velocity, ${ }^{\mathcal{B}_{k}} \mathbf{v}_{j / k}$, using the transport equation:

$$
\frac{{ }^{\mathcal{I}} d}{d t}\left(\mathbf{r}_{j / k}\right)={ }^{\mathcal{B}_{k}} \frac{d}{d t}\left(\mathbf{r}_{j / k}\right)+{ }^{\mathcal{I}} \boldsymbol{\omega}^{\mathcal{B}_{k}} \times \mathbf{r}_{j / k} .
$$

In matrix notation,

$$
\left[{ }^{\mathcal{I}} \mathbf{v}_{j / k}\right]_{\mathcal{B}_{k}}=\left[{ }^{\mathcal{B}_{k}} \mathbf{v}_{j / k}\right]_{\mathcal{B}_{k}}+\left[\omega_{k} \mathbf{z}_{k} \times \mathbf{r}_{j / k}\right]_{\mathcal{B}_{k}}
$$

Using $\mathbf{r}_{j / k}=x_{j / k} \mathbf{x}_{k}+y_{j / k} \mathbf{y}_{k}$ yields

$$
\left[\begin{array}{c}
\cos \left(\theta_{j}-\theta_{k}\right)-1 \\
\sin \left(\theta_{j}-\theta_{k}\right)
\end{array}\right]_{\mathcal{B}_{k}}=\left[\begin{array}{c}
\dot{x}_{j / k} \\
\dot{y}_{j / k}
\end{array}\right]_{\mathcal{B}_{k}}+\omega_{k}\left[\begin{array}{c}
-y_{j / k} \\
x_{j / k}
\end{array}\right]_{\mathcal{B}_{k}} .
$$

Solving for $\theta_{j}-\theta_{k}$ yields

$$
\theta_{j}-\theta_{k}=\arctan \left(\frac{\dot{y}_{j / k}+\omega_{k} x_{j / k}}{1+\dot{x}_{j / k}-\omega_{k} y_{j / k}}\right) .
$$

With this relationship, calculating particle $j$ 's velocity orientation relative to $k$ requires knowledge of $k$ 's turning rate as well as the position and velocity of particle $j$ with respect to $k$. Assuming the relative position, $\mathbf{r}_{j / k}$, and turning rate, $\omega_{k}$, is measured, each particle can estimate the relative velocity, ${ }^{\mathcal{B}_{k}} \mathbf{v}_{j / k}$, in the path frame, $\mathcal{B}_{k}$, using the estimator described next.

\section{B. Velocity Estimation}

Consider the case where particle $k$ is estimating the relative velocity of particle $j$ in frame $\mathcal{B}_{k}$. In this case, let $\hat{\mathbf{r}}_{j / k}=\hat{x}_{j / k} \mathbf{x}_{k}+\hat{y}_{j / k} \mathbf{y}_{k}$ and ${ }^{\mathcal{B}_{k}} \hat{\mathbf{v}}_{j / k}=\dot{\hat{x}}_{j / k} \mathbf{x}_{k}+\dot{\hat{y}}_{j / k} \mathbf{y}_{k}$ be the position and velocity estimates, respectively. Also, let $\triangle \mathbf{r}_{j / k} \triangleq \hat{\mathbf{r}}_{j / k}-\mathbf{r}_{j / k}$ and $\triangle^{\mathcal{B}_{k}} \mathbf{v}_{j / k} \triangleq \mathcal{B}_{k} \hat{\mathbf{v}}_{j / k}-{ }^{\mathcal{B}_{k}} \mathbf{v}_{j / k}$ represent the estimation errors for position and velocity, respectively. Note that we estimate the velocity of particle $j$ with respect to particle $k$ in frame $\mathcal{B}_{k}$. Choosing the estimator dynamics

$$
\begin{aligned}
& { }^{\mathcal{B}_{k}} \frac{d}{d t}\left(\hat{\mathbf{r}}_{j / k}\right)=-K_{1} \triangle \mathbf{r}_{j / k}+{ }^{\mathcal{B}_{k}} \hat{\mathbf{v}}_{j / k} \\
& { }^{\mathcal{B}_{k}} \frac{d}{d t}\left(\hat{\mathbf{v}}_{j / k}\right)=-K_{2} \triangle \mathbf{r}_{j / k},
\end{aligned}
$$

where $K_{1}>0$ and $K_{2}>0$, yields the following error dynamics:

$$
\begin{aligned}
\underbrace{\frac{\mathcal{B}_{k} d}{d t}\left[\begin{array}{c}
\triangle \mathbf{r}_{j / k} \\
\triangle^{\mathcal{B}_{k}} \mathbf{v}_{j / k}
\end{array}\right]_{\mathcal{B}_{k}}}_{\triangleq \dot{e}_{j / k}} & =\underbrace{\left[\begin{array}{cc}
-K_{1} & 1 \\
-K_{2} & 0
\end{array}\right]}_{\triangleq A} \underbrace{\left[\begin{array}{c}
\triangle \mathbf{r}_{j / k} \\
\triangle^{\mathcal{B}_{k}} \mathbf{v}_{j / k}
\end{array}\right]_{\mathcal{B}_{k}}}_{\triangleq e_{j / k}} \\
& +\underbrace{\left[\begin{array}{c}
0 \\
-\mathcal{B}_{k} \mathbf{a}_{j / k}
\end{array}\right]_{\mathcal{B}_{k}}}_{\triangleq g_{j / k}(t)} .
\end{aligned}
$$

When written in this form, we see the estimator is a linear system $\dot{e}_{j / k}=A e_{j / k}+g_{j / k}(t)$, where $g_{j / k}(t)$ is a perturbation.

Representing the equations in vector notation is useful in order to study the stability of the system, but the secondorder model (5) and relative orientation relationship (13) utilize Cartesian coordinates with respect to the frame $\mathcal{B}_{k}$. To be consistent, we can rewrite (14) as

$$
\begin{aligned}
&{ }^{\mathcal{B}_{k}} \frac{d}{d t}\left(\hat{x}_{j / k}\right)=-K_{1} \triangle x_{j / k}+\dot{\hat{x}}_{j / k} \\
&{ }^{\mathcal{B}_{k}} \frac{d}{d t}\left(\hat{y}_{j / k}\right)=-K_{1} \triangle y_{j / k}+\dot{\hat{y}}_{j / k} \\
&{ }^{\mathcal{B}_{k}} \frac{d}{d t}\left(\dot{\hat{x}}_{j / k}\right)=-K_{2} \triangle x_{j / k} \\
&{ }^{\mathcal{B}_{k}} \frac{d}{d t}\left(\dot{\hat{y}}_{j / k}\right)=-K_{2} \triangle y_{j / k},
\end{aligned}
$$

where $\triangle x_{j / k} \triangleq \hat{x}_{j / k}-x_{j / k}$ and $\triangle y_{j / k} \triangleq \hat{y}_{j / k}-y_{j / k} . \hat{x}_{j / k}$ and $\hat{y}_{j / k}$ represent the $x$ and $y$ position estimates, whereas 
$\dot{\hat{x}}_{j / k}$ and $\dot{\hat{y}}_{j / k}$ represent the $x$ and $y$ velocity estimates, respectively.

Lemma 1: The error in the velocity estimation due to the perturbation, $g_{j / k}(t)$, can be reduced by choosing the gains $K_{1}>0$ and $K_{2}>0$ such that the quantity

$$
\frac{K_{1}}{K_{2}}+\frac{K_{2}+1}{K_{1} K_{2}}>0
$$

is minimized.

Proof: Consider the following Lyapunov function

$$
V=e^{T} P e
$$

where $e \triangleq\left[\begin{array}{ll}\triangle \mathbf{r}_{j / k} & \triangle^{\mathcal{B}_{k}} \mathbf{v}_{j / k}\end{array}\right]^{T}$. The matrix $P$ is chosen by solving the Lyapunov equation

$$
P A+A^{T} P=-Q
$$

where $Q \in \mathbb{R}^{2 x 2}$ is the identity matrix. For this system,

$$
P=\left[\begin{array}{cc}
\frac{K_{2}+1}{2 K_{1}} & -\frac{1}{2} \\
-\frac{1}{2} & \frac{K_{2}+K_{1}^{2}+1}{2 K_{1} K_{2}}
\end{array}\right] \text {. }
$$

Taking the derivative with respect to time yields

$$
\begin{aligned}
\dot{V}= & -e^{T} Q e \\
& -{ }^{\mathcal{B}_{k}} \mathbf{a}_{j / k}\left(-\triangle \mathbf{r}_{j / k}+\left(\frac{K_{1}}{K_{2}}+\frac{K_{2}+1}{K_{1} K_{2}}\right) \triangle^{\mathcal{B}_{k}} \mathbf{v}_{j / k}\right) .
\end{aligned}
$$

The estimator assumes that the relative position is known; therefore, the error in the position estimate is negligible. As a result, (21) ensures $\dot{V} \leq 0$ for $\|e\| \geq b$, where $b$ is proportional to $\left(\frac{K_{1}}{K_{2}}+\frac{K_{2}+1}{K_{1} K_{2}}\right)\left\|g_{j / k}(t)\right\|_{\mathcal{L}}$. Therefore, minimizing (17) will reduce the lower bound $b$.

\section{Observer-Based Feedback Control}

Let's now consider an $N$-particle system that obeys the second-order model (5). Each particle utilizes the estimator (16) to determine the relative velocities of the other particles. These estimates are then used to calculate the relative orientations of the particles using (13). Finally, each particle implements the desired control using the relative orientations, $\hat{\boldsymbol{\theta}}$. The state-space representation of the combined system is:

$$
\begin{aligned}
\dot{x}_{k} & =\cos \left(\theta_{k}\right) \\
\dot{y}_{k} & =\sin \left(\theta_{k}\right) \\
\dot{\theta}_{k} & =\omega_{k} \\
\dot{\omega}_{k} & =K_{p}\left(\hat{u}_{k}-\omega_{k}\right) \\
{ }^{\mathcal{B}_{k}} \dot{\hat{x}}_{j / k} & =-K_{1} \triangle x_{j / k}+\dot{\hat{x}}_{j / k} \\
{ }^{\mathcal{B}_{k}} \dot{\hat{\hat{y}}}_{j / k} & =-K_{1} \triangle y_{j / k}+\dot{\hat{y}}_{j / k} \\
{ }_{\mathcal{B}_{k}} \ddot{\hat{x}}_{j / k} & =-K_{2} \triangle x_{j / k} \\
{ }^{\mathcal{B}_{k}} \ddot{\hat{y}}_{j / k} & =-K_{2} \triangle y_{j / k},
\end{aligned}
$$

with $k, j=1, \ldots, N$ and $\hat{u}_{k}$ represents the desired control law.

Let

$$
\widehat{\theta_{j}-\theta_{k}}=\arctan \left(\frac{\dot{\hat{y}}_{j / k}+\omega_{k} \hat{x}_{j / k}}{1+\dot{\hat{x}}_{j / k}-\omega_{k} \hat{y}_{j / k}}\right) .
$$

and $\hat{\boldsymbol{\theta}}=\left[{\widehat{\theta_{1}-\theta_{k}}}_{1}, \ldots,{\widehat{\theta_{N}-\theta_{k}}}\right]$. Note that the combination of the control law and estimator further define the perturbation in (15) as a vanishing perturbation ${ }^{1}$ because the particles do not move in the body frame, $\mathcal{B}_{k}$, when converged to the desired formation. Since the particles are stationary, they have velocity and acceleration, ${ }^{\mathcal{B}_{k}} \mathbf{a}_{j / k}$, equal to zero.

For a parallel formation, $\hat{u}_{k}=\phi_{k}(\hat{\boldsymbol{\theta}})$ in (22). Noting that the parallel control law is a summation of sine terms and the relative orientation calculation uses an inverse tangent, the control law can be simplified using trigonometric identities to

$$
\phi_{k}(\hat{\boldsymbol{\theta}})=-\frac{K}{N} \sum_{j=1}^{N} \frac{\dot{\hat{y}}_{j / k}+\omega_{k} \hat{x}_{j / k}}{\sqrt{\left(\dot{\hat{y}}_{j / k}+\omega_{k} \hat{x}_{j / k}\right)^{2}+\left(1+\dot{\hat{x}}_{j / k}-\omega_{k} \hat{y}_{j / k}\right)^{2}}} .
$$

Implementation of the circular control law is achieved the same way using $\hat{u}_{k}=\psi_{k}(\mathbf{r}, \hat{\boldsymbol{\theta}})$ in (22). Note that the relative orientation is used to calculate the centers of rotation (3) in particle $k$ 's path frame.

Proposition 2: The observer-based feedback control algorithm described in (22) will stabilize the desired formation as long as each particle's turning rate and distance between particles remains sufficiently small.

These conditions on the turning rate and distance come from the perturbation term, $g_{j / k}(t)$. As shown in (15), the perturbation is a function of the acceleration, ${ }^{\mathcal{B}_{k}} \mathbf{a}_{j / k}$. When derived, this term depends on the particle's angular rate, angular acceleration, relative velocity orientation, and relative position. By reducing the distance between particles and their turning rates, the acceleration term decreases, which allows for more accurate estimations. With accurate estimates of the relative velocity orientation, the particles converge to the desired formation.

The state-space representation of the system has been programmed to simulate the behavior of a group of particles. Fig. 4 shows a simulation of the parallel control law, while Fig. 5 displays a simulation of the circular control law. Both simulations begin with each particle being given a random velocity orientation, a random position, and zero turning rate. Each particle also maintains $N-1$ estimates for the states of the other particles as shown in (22). The particle's position estimates are initialized to their actual position in the body frame, whereas the particle's velocity estimates are initialized to zero.

The plots for each simulation show the error in the position and velocity estimates for each particle. There are $(N-1)^{2}$ estimates shown because each individual particle has $N-1$ estimators. Also, note that the error in the estimates approaches zero as $t \rightarrow \infty$, which implies that each particle determines the position and velocity of the other particles as time goes to infinity.

\section{UNDERWATER VEHICLE TESTBED}

The control laws and estimator have been designed using an idealized modeling framework. This technique allows higher level control laws to be studied for stability and convergence without the need for a specific system model to be utilized. However, these control laws need to be applied

\footnotetext{
${ }^{1} \mathrm{~A}$ vanishing perturbation is an additional term to a system that evaluates to zero when the state of the system is at equilibrium [4].
} 

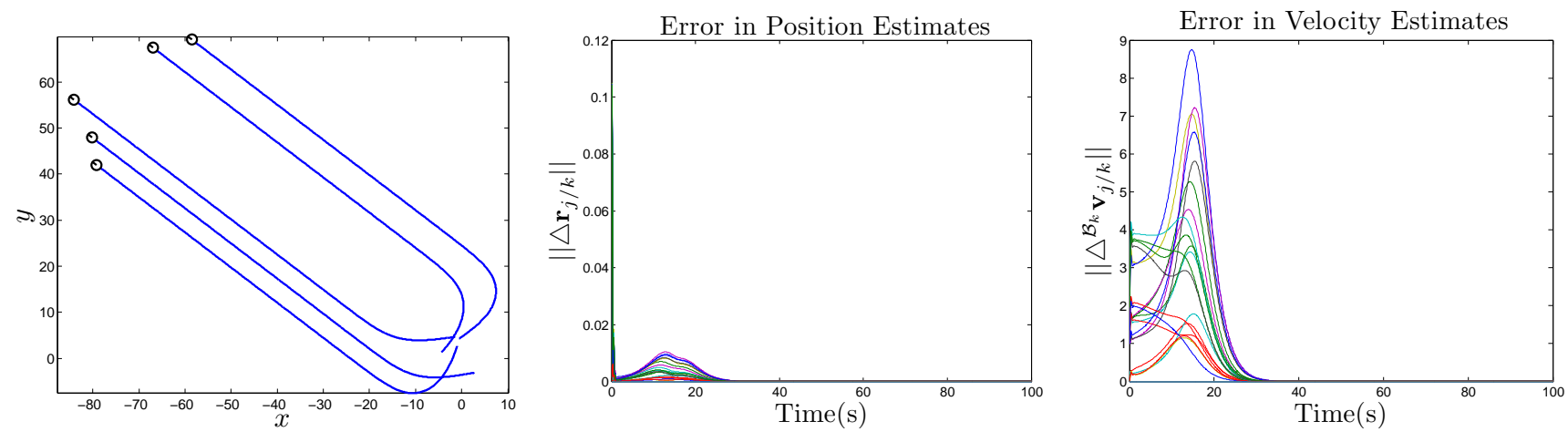

Fig. 4. A simulation of five self-propelled particles performing the parallel control law while estimating the other's relative velocity. Each particle is given a random starting position and velocity with control gains $K=-1, K_{p}=1, K_{1}=10$, and $K_{2}=100$.
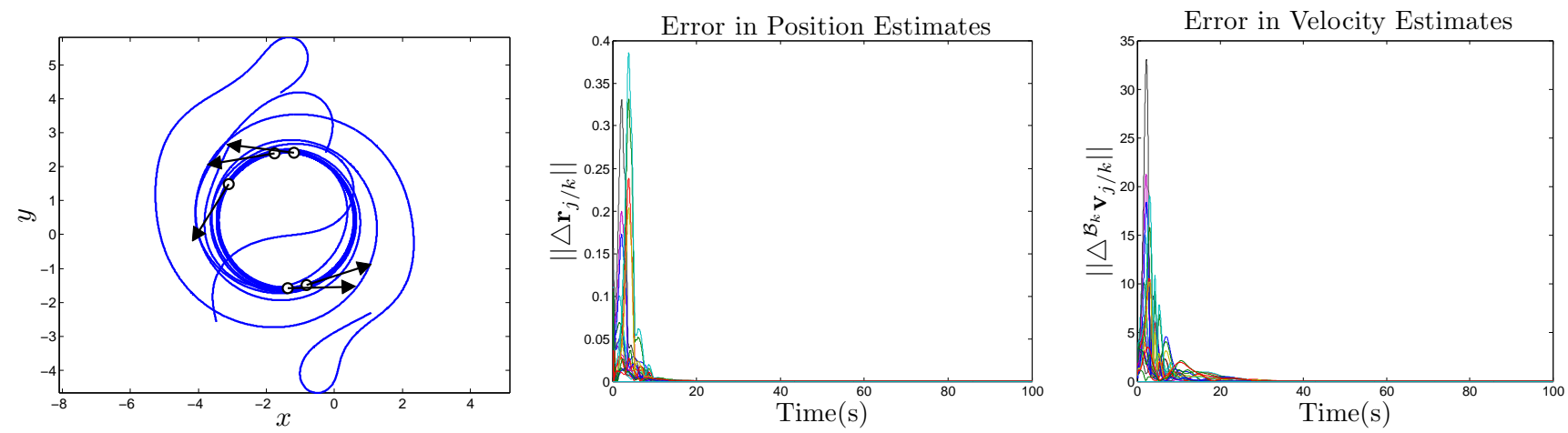

Fig. 5. A simulation of five self-propelled particles performing the circular control law using their individual position and velocity estimates with $\omega_{0}=.5$. Each particle is given a random starting position and velocity with control gains $K=1, K_{p}=1, K_{1}=10$, and $K_{2}=100$.

to a physical system to demonstrate the usefulness of the control law.

Taking this point into consideration, we are developing a submarine testbed for experimental validation of motion coordination algorithms. Each submarine is a radio-controlled 1:60 scale model of the U.S.S. Albacore and utilizes an onboard microprocessor and sensors to steer the vehicle; Fig. 6 indicates the current state of the testbed. (Additional details are available in [10].) The microprocessor onboard each vehicle serves two separate purposes. First, pitch and yaw rates are stabilized to a desired rate via state-feedback provided by gyroscopes fixed to the vehicle. Secondly, the microprocessor serves as a wireless receiver for updated pitch and yaw rates.

The pitch and yaw rates are updated using an underwater motion capture system shown in Fig. 7. This system is able to track the position and orientation of multiple rigid bodies in real time. We utilize this knowledge to compute the desired yaw rate from (2) or (4). The desired pitch rate is determined by a proportional control to stabilize the submarine's depth. These desired rates are then transmitted to each submarine where the onboard microprocessor takes control of the submarine.

Tests using a single submarine have been used to validate the second-order model under parallel (6) and circular (7) control algorithms without estimation. These tests ensure that the particle model adequately describes the rotational dynamics of a submarine and is sufficient to test the estimation algorithm. Alongside the experimental data is a simulated version with the same initial conditions for comparison.

Fig. 8 displays a single submarine performing the parallel control law with a virtual particle traveling along the positive $x$ axis. The submarine is able to reorient itself in the general direction of the formation before reaching the end of the tank. The experimental result is similar to the simulated result, but converges slower because the turning rate is limited by the submarine's dynamics.

Fig. 9 shows a single submarine performing the circular control law in the tank with a virtual particle. The submarine is circling the correct position, but does not achieve the desired radius during the test.

\section{CONCLUSION}

This paper describes an observer-based feedback control algorithm to stabilize parallel and circular formations using a second-order particle model. Simulations illustrate the results by reproducing the desired motion as well as relative velocity errors that approach zero as time goes to infinity. A submarine testbed is also described and will be used to verify these algorithms on a physical system.

In ongoing research, we are formally analyzing the convergence of the observer-based feedback control algorithm 

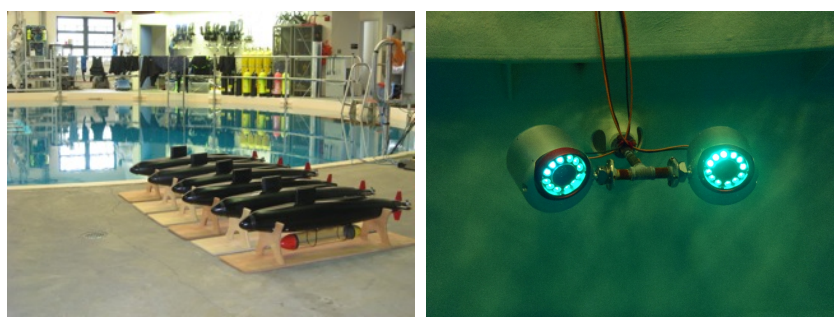

Fig. 6. The underwater vehicle testbed consists of six submarines that operate in the Neutral Buoyancy Research Facility at the University of Maryland and twelve underwater cameras used for motion tracking.
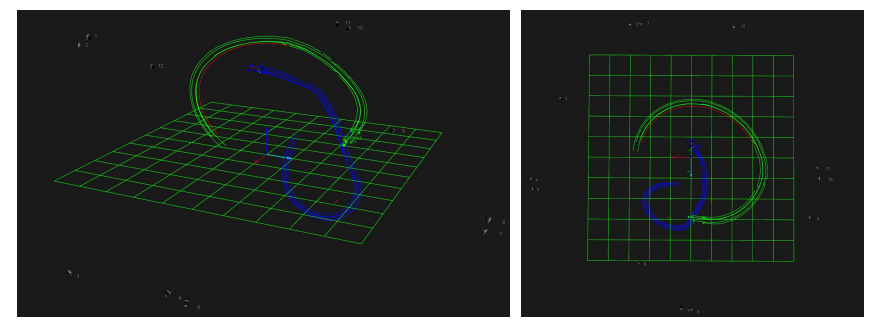

Fig. 7. The motion capture system's runtime environment allows data to be recorded and analyzed.

(Proposition 2). Real-time application of the parallel and circular control laws is being implemented on the experimental testbed. The control algorithms will be examined for their ability to achieve desired motions using the underwater motion-capture system.

\section{REFERENCES}

[1] D. Benedetelli, N. Ceccarelli, A. Garulli, and A. Giannitrapani. Experimental validation of collective circular motion for nonholonomic multi-vehicle systems. Robotics and Autonomous Systems, 58(8):1028-1036, August 2010.

[2] Yongcan Cao, Wei Ren, and Ziyang Meng. Decentralized finite-time sliding mode estimators and their applications in decentralized finitetime formation tracking. Systems and Control Letters, 59(9):522-529, 2010.

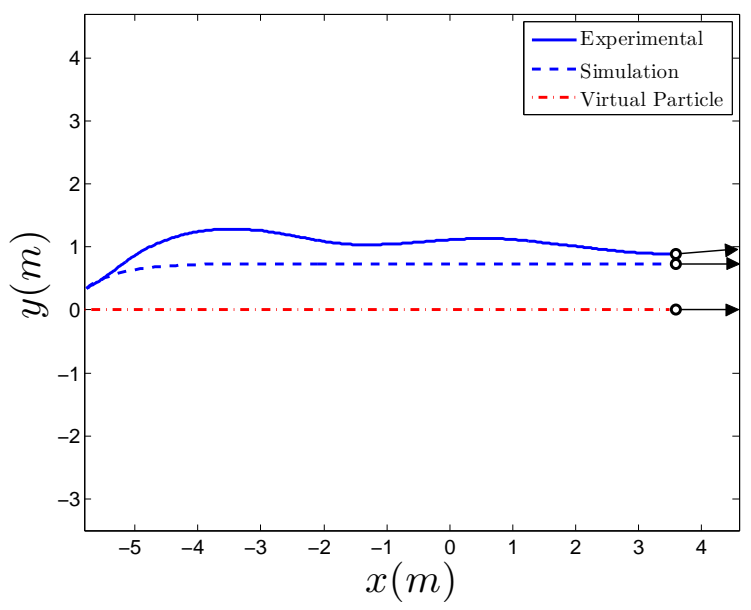

Fig. 8. An experimental run of a single submarine performing the parallel control law with a virtual particle traveling along the $x$ direction. A simulated version of the control law is provided for comparison.

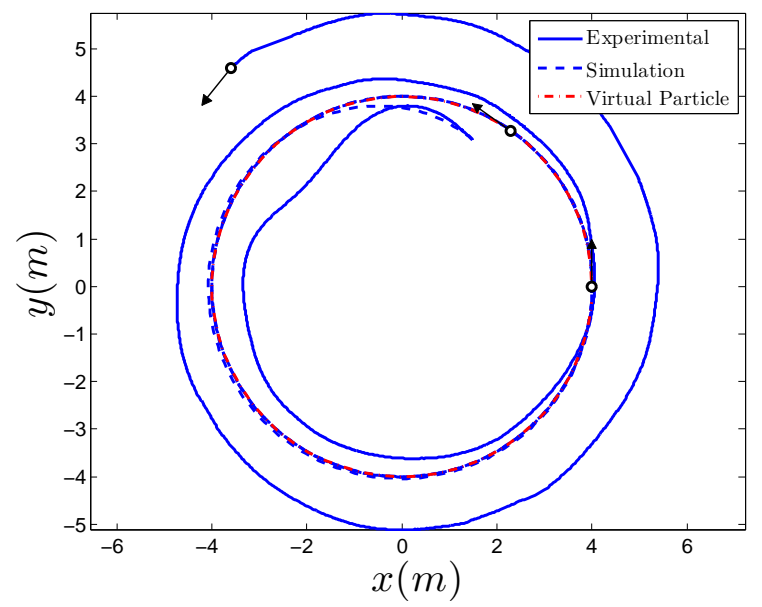

Fig. 9. An experimental run of a single submarine performing the circular control law with a virtual particle. A simulated version of the control law is provided for comparison.

[3] Wei Ding, Gangfeng Yan, and Zhiyun Lin. Collective motions and formations under pursuit strategies on directed acyclic graphs. Automatica, 46(1):174 - 181, 2010.

[4] H. Khalil. Nonlinear Systems. Prentice Hall, Upper Saddle River, NJ 07458, 3rd. edition, 2002.

[5] R. Mellish, S. Napora, and D. A. Paley. Backstepping control design for motion coordination of self-propelled vehicles in a flowfield. International Journal of Robust and Nonlinear Control, 21: n/a. doi:10.1002/rnc. 1702 .

[6] D. A. Paley and C. Peterson. Stabilization of collective motion in a time-invariant flowfield. Journal of Guidance, Control, and Dynamics, 32(3):771-779, May-June 2009.

[7] R. Sepulchre, D. A. Paley, and N. Leonard. Stabilization of planar collective motion: All-to-all communication. IEEE Transactions on Automatic Control, 52(5):811-824, May 2007.

[8] R. Sepulchre, D. A. Paley, and N. E. Leonard. Stabilization of planar collective motion with limited communication. IEEE Transactions on Automatic Control, 53(3):706-719, April 2008.

[9] Housheng Su, Xiaofan Wang, and Zongli Lin. Flocking of multiagents with a virtual leader. IEEE Transactions on Automatic Control, 54(2):293-307, February 2009.

[10] N. Sydney, S. Napora, and D. A. Paley. A multi-vehicle testbed for underwater motion coordination. Proc. 2010 Workshop on the Performance Evaluation for Intelligent Systems, Baltimore, Maryland, September 2010. 